\title{
Nuevas formas comerciales de gran superficie en la Comunidad de Madrid.
}

\author{
Gloria Lora-Tamayo D'Ocon * \\ ASUNCIÓN RIOS CLEMENTE ** \\ Maria Isabel Bodega Fernandez ${ }^{\star \star \star}$ \\ Maria Teresa palacios Estremera **** \\ Adelaida Checa SANCHEZ ${ }^{\star \star \star \star \star}$
}

En todos los paises occidentales, y en España con algunos años de retraso, el comercio ha ido adaptándose a los cambios originados en los estilos de vida de la sociedad. La pequeña tienda de barrio, la de toda la vida, ha dado paso a grandes superficies comerciales que ofrecen en general, variedad, calidad, precios competitivos y una forma de vender atractiva.

Entre los primeros comercios que se establecen en grandes superficies se cuentan los Grandes Almacenes, de creación francesa y los Almacenes Populares, de creación americana y relanzamiento francés, que supusieron a fines de siglo pasado y principios del actual, una revolución comercial por lo que significaron de desespecialización, selección de productos y presentación de las mercancias por primera vez a la vista y tacto del público, además de por la amplia superficie que ocupaban. En nuestro país, y más concretamente en Madrid, ambas fórmulas no consiguen una implantación estable hasta los años cuarenta, fecha a partir de la cual se desarrollan siguiendo trayectorias diferentes:

Los Grandes Almacenes buscan localizaciones centrales, cuentan con

* Universidad Complutense de Madrid.

** FUNDICOT.

*** CSIC.

**** Universidad Complutense de Madrid.

***** Universidad Complutense de Madrid. 
pocos establecimientos, pero las superficies van progresivamente en aumento, llegando en algunos casos a superar los $30.000 \mathrm{~m} 2$ de venta. En la actualidad dos cadenas tienen prácticamente el monopolio de los grandes almacenes en Madrid. Sus locales se hallan en las áreas comerciales consagradas de la ciudad, siendo el polo de atracción de las mismas; únicamente, dos establecimientos escogen localizaciones periféricas, siguiendo una nueva estrategia de implantación dentro de los Centros Comerciales de nueva creación.

Los Almacenes Populares, por su parte, con una superficie media muy inferior a la de los Grandes Almacenes, tuvieron un gran desarrollo en los años 60 , siendo la clave de su éxito los precios económicos. Su implantación en los barrios más populares de la ciudad de Madrid, tanto del centro como de la periferia, así como en algunos municipios de la comunidad, fue reflejo de la clientela a la que servian. Tras un periodo de apogeo en el que proliferan cadenas -algunas de las cuales contaron con un buen número de sucursales, tanto en Madrid, como distribuidas por el resto de España-, desde mediados de la década de los 70, y de forma más clara en la década de los 80 , declinan, reduciéndose considerablemente su número e importancia.

La Comunidad de Madrid cuenta también desde principios de nuestro siglo, con otro tipo de grandes superificies comerciales: se trata de los Mercados de abastos, comercios agrupados polivalentes, formados por agregación de comercios estanco, dedicados a la venta de productos alimenticios, que siguen el principio de economizar los desplazamientos del comprador y favorecer la competencia. El mismo principio siguen las Galerias de Alimentación, de creación posterior, cuya diferencia con el Mercado es la de hallarse situadas en la planta baja de un inmueble dedicado a otros usos, en lugar de ocupar un edificio singular. Unos y otras se hallan distribuidos por toda la geografia madrileña, localizándose los Mercados en las áreas más antiguas de la ciudad y en los municipios más populosos, mientras las galerias se asientan preferentemente en los distritos periféricos de Madrid.

En las dos últimas décadas asistimos a una transformación radical en la estructura comercial de nuestra comunidad, con la proliferación de las grandes superficies localizadas fuera de las zonas comerciales consagradas de la ciudad de Madrid, en su área metropolitana y en los municipios de la periferia, fundamentalmente. La importante expansión metropolitana, con la fuerte demanda de estas áreas, los cambios operados en los modos de vida y en los hábitos de compra del consumidor, asi como la disponibilidad de suelo y su precio más asequible, han sido factores decisivos en el proceso seguido. 
Los dos tipos comerciales que protagonizan esta revolución son el $\mathrm{Hi}-$ permercado y el Centro Comercial. Su origen, estructura y evolución, ofrecen un interés singular.

En este artículo analizamos ambas fórmulas comerciales, tal y como se presentan en el CAM, estudiando también su distribución espacial y los factores de su localización, asi como su impacto en la trama urbana.

\section{1) EL HIPERMERCADO}

El Hipermercado se define como un establecimiento detallista en régimen de autoservicio, con una superficie de venta superior a los 2.500 $\mathrm{m} 2$, ofreciendo un amplio surtido de artículos de alimentación (como mínimo el 60 por 100) y de consumo corriente (textil y bazar) y disponiendo de un gran aparcamiento para la clientela. Por razones de tamaño - tanto en relación con la superficie de venta, como la de almacenaje y aparcamiento- su localización se desplaza a las afueras de las ciudades, dependiendo la rentabilidad del hipermercado de su emplazamiento dentro de un área de atracción de entre 70.000 y 90.000 personas y contando con un radio de acción de 10 a $20 \mathrm{Km}$.

Por su localización, el cliente del hiper necesita desplazarse en automóvil, empleando para ello un tiempo variable, que puede situarse en torno a los 20 minutos, una de las razones que motivan que las visitas a estos establecimientos se espacien en el tiempo, efectuándose en ellos las compras fuertes. Sus caracteristicas fundamentales son el amplio surtido, buena calidad y bajos precios de los productos, asi como el tener un horario comercial continuado, por lo general de 10 a 22 horas. El perfil medio del comprador del hipermercado es un «ama de casa joven, que trabaja fuera del hogar, con status medio-alto, vivienda confortable con congelador independiente y uso de tarjetas de crédito, y residente en poblaciones mayores de 50.000 habitantes" (D/A oct. 1987, p. 127).

El Hipermercado es una fórmula francesa, creada por los comerciantes Marcel Fournier y los hermanos Jacques y Denis Defforey, quienes en 1963 abrieron en las afueras de Paris (St. Geneviève des Bois) un establecimiento con una superficie de venta de $2.600 \mathrm{~m} 2$ ( $4.000 \mathrm{~m} 2$ edificados) y 18 cajas de salida rápida, en el que aplicaban la técnica del discount al régimen de autoservicio. De aquí nacería la cadena francesa de hipermercados "Carrefour", cuya expansión por el resto de Francia y Europa ha sido de notable importancia.

Con el tiempo, el hipermercado ha modificado ligeramente su estruc- 
tura, adquiriendo mayor importancia los productos no alimenticios, creando marcas propias y habilitando una galeria anexa de comercios detallistas especializados y servicios, sobre todo de restauración. Esta simbiosis hipermercado-pequeños establecimientos especializados, rentable por ambas partes, ha conducido en los últimos años a la instalación de hipermercados dentro de Centros Comerciales de amplias dimensiones, como «locomotoras» o focos de atracción de clientela de los mismos.

El ritmo de crecimiento del hipermercado en España ha sido vertiginoso. Entre 1973, fecha en que se abre el primero de estos establecimientos en nuestro país (PrYCA de Prat de Llobregat, Barcelona) hasta 1990, han nacido 90 hipermercados, siendo en esta última fecha su superficie media de $7.044 \mathrm{~m}^{2}$ y contando por término medio con $210 \mathrm{em}$ pleados, 40 cajas y 1.100 plazas de parking, por establecimiento.

El primer hipermercado madrileño fue Jumbo, al Norte de la ciudad, que abrió sus puertas en junio de 1975. Desde entonces hasta 1991 se han inaugurado 17 más en el conjunto de la Comunidad, siendo su localización fundamentalmente periurbana: únicamente cinco se sitúan dentro del municipio de Madrid. De los restantes, la mayoria se hallan al Sur, en el área más poblada del territorio, dentro de los municipios de Alcorcón, Getafe, Leganés, Móstoles y Parla; Fuenlabrada contó también con un hipermercado (Preco), que sufrió un siniestro por incendio en 1989 y ha reanudado su actividad recientemente. Al Este, San Fernando y Alcalá de Henares cuentan con sendos hipermercados. Al Norte, únicamente Alcobendas. Por último, la periferia occidental metropolitana figura como el área pionera en la implantación de hipermercados - si descontamos Jumbo- con un establecimiento en Majadahonda, abierto en 1976 y otro en las Rozas, que data de 1981. Los restantes hipermercados se han abierto en la década de los 80 o al inicio de los 90 .

La distribución espacial de los establecimientos sigue claramente la de la población: así, los municipios que cuentan con hiper son los que aglutinan un mayor volumen de residentes. Por otra parte, el emplazamiento de los centros, fundamentalmente en la periferia próxima de $\mathrm{Ma}$ drid, amplia la clientela potencial de los mismos, dando servicio - y favoreciéndose con ello- a los distritos exteriores de la ciudad.

Las empresas más potentes en el ámbito del hipermercado son Pryca, Continente, Alcampo e Hipercor. Las tres primeras son de capital francés, mientras Hipercor - filial de la cadena de Grandes Almacenes «El Corte Inglés»- es la única cuyo capital es enteramente español. La representación de estas empresas en la CAM es variable: en el interior de la ciudad predomina Alcampo - filial de la francesa Auchan-, en cuanto a número de centros; fuera de ella, la empresa cuenta con establecimientos 
en Getafe, Leganés y Alcalá de Henares, donde funcionan como «locomotoras" de Centros Comerciales. Próximamente abrirá un nuevo establecimiento en Alcorcón. Pryca - Promotora de Hipermercados, S. A., constituida por las sociedades francesas Simago y Carrefour- es hoy en dia la primera empresa de Hipermercados en España. Cuenta en la comunidad con centros en Majadahonda, Las Rozas y San Fernando de $\mathrm{He}-$ nares, a los que ha agregado galerias comerciales. Continente - perteneciente a la sociedad española Saudisa, creada por el grupo francés de distribución comercial Promodés - tiene un centro en Alcobendas, que figuraba hasta hace poco como uno de los hipermercados mayores de España. La misma empresa está construyendo un nuevo centro en Móstoles. Hipercor es la primera empresa de hipermercados de capital español. Su centro de Alcorcón, abierto en 1989, es el mayor de la comunidad, con cerca de $15.000 \mathrm{~m}^{2}$ de superficie de venta al público y anexo a una galería de «tiendas Corty». Próximamente se abrirá un nuevo centro Hipercor en la calle Méndez Álvaro.

El resto de los hipermercados de la CAM no pertenecen a grandes cadenas y tienen, en general, dimensiones más reducidas.

No queremos dejar de citar dos establecimientos de comercio mixto de grandes dimensiones (en torno a $10.000 \mathrm{~m}^{2}$ ), que no son hipermercados en el sentido estricto y que nacieron como establecimientos mayoristas: se trata de los dos centros "MAKRO" de Leganés y el Polígono "Las Mercedes" (Madrid), que posiblemente por competencia con los hipermercados - donde se proveen en la actualidad multitud de pequeños comerciantes-, han extendido su carnet de compra a muchos colectivos; el localizado en Leganés, concretamente, ha iniciado recientemente una nueva etapa abriendo por completo sus puertas al público, por lo que debe ser incluido entre los hipermercados.

Por último señalar la existencia de otra serie de establecimientos que, a pesar de tener una superficie de venta superior a $2.500 \mathrm{~m}^{2}$, no son considerados hipermercados, por la específica orientación de su comercio a hogar-limpieza y alimentación, como Mercadona o Eco, por ejemplo.

\section{LOS CENTROS COMERCIALES}

Se denomina Centro Comercial al "conjunto de establecimientos con una oferta de productos y servicios diversificada, que se proyectan dentro de unas instalaciones operadas como una sola unidad de gestión" (I Congreso Nacional de Centros Comerciales, octubre 1988). Las caracteristicas fundamentales que definen un Centro Comercial son la unidad de gestión, 
el asentarse sobre una superficie minima de $2.500 \mathrm{~m}^{2}$ y albergar al menos a 15 comerciantes. El tope máximo no se determina, contando los centros mayores con superficies en torno a los $100.000 \mathrm{~m}^{2}$ y agrupando a más de 300 comerciantes.

El fenómeno del Centro Comercial, que obedece a la necesidad de concentrar en un punto determinado un número elevado de comercios diversificados y servicios comerciales, sobre todo de restauración y ocio, aparece en España en los años 70 , aunque no cobra fuerza hasta los 80 , con un cierto desfase respecto a los Estados Unidos y Europa Occidental. El desfase viene motivado por la mayor lentitud en la evolución de los hábitos de compra de la población. Éstos son reflejo, a su vez, de los cambios en las formas de vida: el aumento de la renta per cápita, la incorporación de la mujer al trabajo y la extensión del automóvil, son factores que inciden en la búsqueda de un equipamiento comercial que posibilite realizar las compras en menos tiempo, con más comodidad, un horario más amplio y una variedad mayor en la oferta. La expansión de las ciudades y la creación de nuevas formas residenciales en la periferia de las mismas, asi como el envejecimiento y saturación de los centros urbanos tradicionales, explican también el desarrollo de los Centros Comerciales.

El origen del Centro Comercial es difícil de precisar, entre otros motivos por los distintos tipos de agrupaciones comerciales que caben en esta denominación: galerias comerciales compuestas por un corto número de establecimientos; centros de mayor superficie, integrados por tiendas de modas; zocos o centros de pequeño o mediano tamaño, que reúnen comercios variados y locales de ocio; Centros Comerciales de mayor envergadura, donde se multiplican los comercios, los locales destinados al ocio y la restauración y una variada oferta de productos alimenticios, denominados en la actualidad "centros integrales"; centros en los que, junto a esta oferta, aparece la cultural o la deportiva, derivando del concepto "Centro Comercial» al de «Parque Comercial», etcétera.

Para S. Pagés (Director de promoción empresarial de la Cámara de Comercio de Barcelona), el primer Centro Comercial del mundo (entendido como de oferta especializada) es el Mercado de iniciativa pública, que acerca la oferta al ciudadano y en torno al cual aparece una actividad comercial complementaria a aquella (ARAL 3, sept. 88). Los zocos de los paises africanos y árabes, por otra parte, son una de las formas de agrupación comercial más antigua conocida.

Los centros de las ciudades, donde la oferta de comercios, servicios y ocio es completa y variada, se hallan, en nuestro criterio, en la base de los modernos Centros Comerciales. La expansión de las ciudades -el desarrollo de urbanizaciones y ciudades satélites en su entorno-, con la 
dificultad de acceso al centro de las mismas, la inseguridad ciudadana y la congestión del tráfico, son factores que inciden en la creación de estructuras que concentren una oferta comercial y de servicios similar a la del centro urbano, como réplica de éste, en puntos concretos alejados de él, pero con una clientela potencial importante. Este es el origen de los "shopping centers" estadounidenses y canadienses, que han tenido una influencia directa en los modernos centros comerciales españoles.

El primer Centro Comercial del mundo se creó en Rolland Park en 1907, aunque los Centros Comerciales realmente planificados y gestionados unitariamente no se desarrollan en Estados Unidos hasta el término de la segunda guerra mundial. En pocos años, la nueva fórmula comercial se divulga por Europa, siendo su introductor el apátrida Robert de Balkany. A partir de 1955 aparecen los primeros centros comerciales en Suecia, Alemania, Inglaterra y Francia.

El modelo americano influirá, a través de Francia, en el tipo de Centro Comercial que va a proliferar en nuestro pais en los años 80: se trata de los centros que integran comercio diversificado, ocio y restauración y en la mayor parte de los casos cuentan con una "locomotora", es decir, un local comercial de grande o mediana superficie, que atrae por si solo tal volumen de clientela que "arrastra" al resto de los comercios integrados en el centro. La locomotora suele ser un gran supermercado o un hipermercado, un almacén popular o un gran almacén, y en muchos casos, dos de estos tipos comerciales.

El primer centro de estas características instalado en Francia - «Elysée 2", 1967- es obra de Robert de Balkany, quien crea un núcleo comercial en un área de verano, donde en una sóla planta de $10.000 \mathrm{~m}^{2}$, a cielo abierto, instala 31 locales de comercio y servicios. El elemento de atracción es una bolera, la primera automática de Europa. Dos años más tarde crea también en Francia «Parly-2» (Versalles), centro de alto nivel, donde en $50.000 \mathrm{~m}^{2}{ }^{2}$ se instalan 128 locales -más tarde se amplía al doble- Estos dos centros constituirán un ejemplo a seguir por algunos de los Centros Comerciales españoles más importantes, como "Baricentro", en Barcelona, o "Madrid-2" en nuestra ciudad.

Con anterioridad a este tipo de centros, existen en nuestro pais y concretamente en la Comunidad de Madrid, otras dos fórmulas que se ajustan plenamente al concepto de Centros Comerciales: se trata de los «Centros de Moda" y los «Zocos".

Realmente los primeros Centros Comerciales madrileños, que datan de la década de los 70 , son más bien centros de moda, agrupación de comercios especializados de vestido, calzado y complementos -asociación comercial de gran fuerza, presente en todos los ejes comerciales 
urbanos-, cuya filosofia, coincidente en gran medida con la de los viejos mercados y galerias de alimentación, es la de la multiplicación de la oferta al cliente, para potenciar la atracción. Se suelen instalar en áreas comerciales del interior de la ciudad, ocupando los bajos y sótanos de un inmueble dedicado a otros usos. A este tipo corresponden los "Multicentro" de Princesa, Serrano y Orense y «Modashopping" de Azca, todos ellos en Madrid. "Las Tiendas de Princesa 40", hoy desaparecido, es otro ejemplo.

Los Zocos constituyen experiencias pioneras en nuestro pais y en la Comunidad de Madrid. El primero de ellos fue diseñado en 1978 y abierto al público en 1979, en Majadahonda. Su nombre, según su creador, Fernando Ramos, se inspiró en el Zoco de Marraquech y en él pretendió unir, por primera vez en España, fuera del casco urbano, lo comercial con lo lúdico. Se trata de edificios singulares, de dos o tres plantas por lo general, donde se agrupan locales comerciales, servicios de restauración y locales recreativos, sobre todo pequeños cines. Se localizan fundamentalmente en los municipios de la periferia de Madrid, dentro de las áreas de reciente expansión, con población joven y nivel de renta medio y alto $y$ donde el equipamiento comercial y recreativo es deficitario.

A estas dos lineas diferentes, habria que añadir la resultante de la evolución de los hipermercados. Las galerías comerciales que desde finales de los años 70 y durante la década de los 80 crean éstos, supone la consagración de la fórmula "pequeños comercios especializados + gran superficie de alimentación» que ha constituido el éxito de los modernos Centros Comerciales que cuentan con al menos una «locomotora".

Se pueden establecer distintas clasificaciones de los Centros Comerciales madrileños, en función de su tamaño, su localización en el interior urbano o en la periferia, su tipologia formal o su especialización. En este apartado nos hemos orientado a trazar a grandes rasgos una tipología de los centros según su superficie. La localización de los mismos viene reseñada en el Cuadro 1, asi como en la cartografia adjunta. En cuanto a sus características formales y estructurales, serán tema de comentario de los próximos apartados.

Los Centros Comerciales madrileños pueden clasificarse, según su tamaño, en:

1.) Centro de Barrio o Distrito, con una superficie bruta alquilable (SBA) de hasta $7.500 \mathrm{~m}^{2}$. Este tipo se extiende por la periferia de Madrid: "Zocos" de la zona Oeste, Burgo Centro de Las Rozas, Centro Comercial Las Rozas, Tutti Centro, Centro Comercial Monteclaro, Centro Comercial El Plantío, Boulevard de la Moraleja, Centro Comercial de Vicálvaro, etc.... Normalmente sirven a una población de entre 5.000 y 50.000 habitantes y 
suelen contar con una "locomotora" especializada de alimentación, que actúa a modo de gancho de la clientela, por su prestigio (Alfaro, Mercadona, Alcampo, etc...) Se habla en estos casos de Centros Comerciales integrales (es decir, con una oferta multivariada). En esta categoria cabria incluir también los centros especializados orientados hacia la moda, el arte o el diseño (Multicentros, Galería del Prado, Mercado Puerta de Toledo) en el casco urbano de Madrid, siempre que cumplan los requisitos anteriormente señalados; se habla entonces de un Centro Comercial Temático.

2.) Centro Intermedio, cuya SBA oscila entre 7.500 y $25.000 \mathrm{~m}^{2}$. Su área de influencia se extiende a una población de entre 50.000 y 150.000 personas. Su principal caracteristica es el predominio de una gran superficie comercial, locomotora por antonomasia, y la existencia de una galería comercial no muy grande, compuesta por comerciantes especializados independientes. Como ejemplo de este tipo en Madrid, podemos mencionar los distintos Prycas y el Continente de Alcobendas.

3.) Centro Regional o Parque Comercial, con una SBA por encima de $25.000 \mathrm{~m}^{2}$ y un potencial de población superior a las 200.000 personas. Estos centros disponen de varias "locomotoras" de gran impacto. Su estrategia se basa fundamentalmente en una oferta muy amplia de ocio: restauración, cines, boleras, incluso, como es el caso de Parque Sur en Leganés, parque de atracciones y parque acuático. Estos Parques Comerciales se sitúan en el exterior de las grandes aglomeraciones urbanas, con zonas despejadas, facilidad de aparcamiento y vias rápidas de comunicaciones. Funcionan como polos de atracción para grandes núcleos de población y pretenden a su vez paliar deficiencias de infraestructura y equipamiento en las áreas donde se instalan. El número de estos grandes centros es mucho más reducido: en Madrid, el ya mencionado Parque-Sur y el centro regional Madrid-2 (La Vaguada). En el resto de España, podemos indicar Baricentro en Barcelona, Nuevo Centro en Valencia, Las Salesas en Oviedo y Cuatro Caminos en La Coruña. Todos ellos superan ampliamente los $25.000 \mathrm{~m}^{2}$ señalados.

\section{DISTRIBUCIONN ESPACIAL Y FACTORES DE LOCALIZACIÓN}

En la fig. 1 se señalan los Centros Comerciales e Hipermecados de la Comunidad de Madrid, en base a la relación que se ofrece en el Cuadro 1. Las fuentes empleadas en su elaboración han sido el estudio sobre "Centros Comerciales y Mercados Minoristas. Informe y directorio", de la Dirección General de Comercio Interior (1991) y la «Guia de Grandes Superficies Comerciales de la Comunidad de Madrid" (1991), de la Conse- 


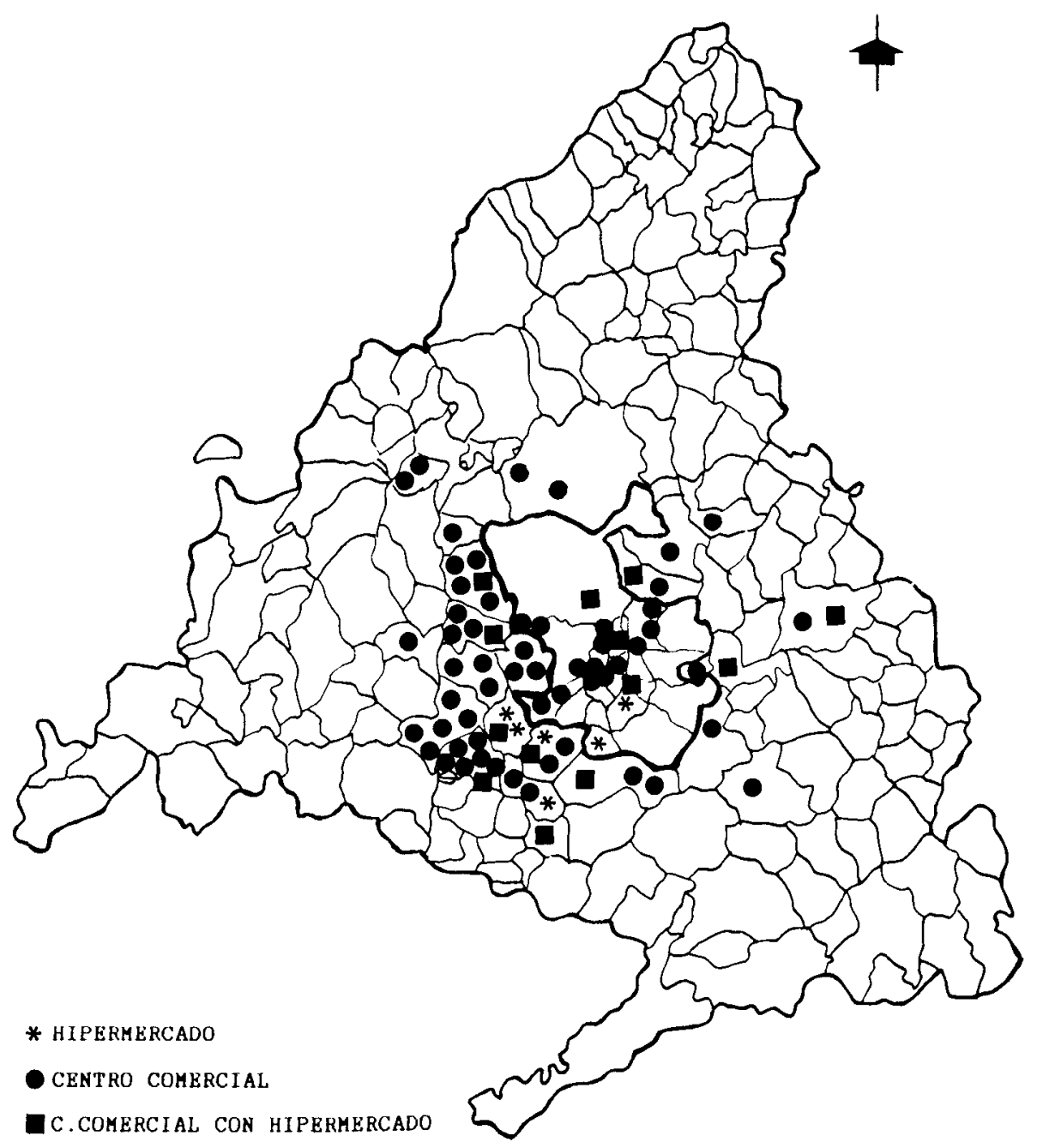

Fig. 1. Hipermercados y Centros Comerciales en la Comunidad de Madrid.

jeria de Economía de la Comunidad de Madrid. La encuesta y el trabajo de campo personales, realizados durante los últimos meses de 1991 y primeros de 1992, han sido métodos fundamentales a la hora de corregir y completar la información de base '. Dada la dificultad de elaboración de

En nuestro listado incluimos únicamente los Centros Comerciales cuya superficie es superior a $2.500 \mathrm{~m}^{2}$ y que tienen unidad de gestión. Asi, hemos excluido algunos centros 
este listado, por el dinamismo en el proceso de creación y transformación de las estructuras comerciales, así como por los problemas en la tipificación de algunos modelos, no consideramos éste concluyente, sino sujeto, igual que las otras fuentes, a involuntarios errores $u$ omisiones ${ }^{2}$.

La distribución espacial de los hipermercados en la CAM ya ha sido señalada con anterioridad: dentro del municipio de Madrid se situan -excepto en el caso de Jumbo (Dto. de Chamartín) - fuera de la almendra, en los distritos periféricos: asi, los diferentes centros Alcampo, en los distritos de Vallecas, Moratalaz y Fuencarral e Hipersur, el centro más pequeño de la serie, en el de Villaverde.

Fuera del municipio de Madrid, los hipermercados se encuentran preferentemente dentro del área metropolitana, en las zonas más pobladas: fundamentalmente el Sur (Alcorcón, Getafe, Leganés, Móstoles y Parla) y el Corredor del Henares (San Fernando y Alcalá). Al Norte, existe un centro en Alcobendas y al Oeste, en las Rozas y Majadahonda.

La localización de los hipermercados, con frecuencia junto a importantes vías de comunicación, es un factor a destacar: asi, Pryca Las Rozas, junto a la Nacional VI, Pryca de San Fernando de Henares y Alcampo de Alcalá de Henares, junto a la carretera de Barcelona, Alcampo Moratalaz, junto a la M-30 o Hipersur, junto a la carretera de Andalucia, por citar algunos ejemplos.

En cuanto a los Centros Comerciales, sólo una pequeña proporción se localiza dentro de la almendra madrileña, tratándose en todos los casos -a excepción de Jumbo con su galería comercial- de centros temáticos. Dentro de ello, los centros de moda (Modashopping, Multicentros, «El Jardin de Serrano"...) escogen siempre localizaciones centrales, situándose en las áreas comerciales consagradas de la ciudad. El «Mercado Puerta de Toledon, centro de arte y diseño dirigido a un público selecto, situado

que recogen las otras fuentes, como Galaxia, Argüelles, Multicentro Pintor, Multicentro Serrano, Centro de Arte y Antigüedades, etc... Tampoco incluimos centros que aún no están en marcha (como Conde Duque) o centros recientemente desaparecidos (Los Sótanos). Recogemos, por el contrario, algunos de reciente creación, como La Dehesa, de Alcalá de Henares, con el hiper Alcampo (junio 1991), El Jardin de Serrano (noviembre 1991) o «Santa Mónica" en Rivas Vaciamadrid (febrero 1992) y otros más antiguos, pero que no se contemplan en las otras fuentes: Cesta, Ecomóstoles y Zoco Coimbra, en Móstoles; Monteclaro, en Pozuelo de Alarcón; El Bosque, Puzzle y Zoco Center, en Villaviciosa de Odón; El Canguro, en Collado Villalba, etc..

${ }^{2}$ En la confrontación de los datos de la Comunidad y del Ministerio de Economia y Hacienda entre si y con los que nos han sido facilitados directamente por algunas entidades. existen importantes divergencias en muchos casos. Por ello, consideramos nuestro listado, igual que los elaborados por aquellos organismos, como un primer fichero de trabajo, a corregir en sucesivas fases. 
también en la almendra, no busca, por el contrario, una localización central, siendo su área de influencia mayor, en función, sobre todo, del carácter restringido y fuerte especificidad de la clientela a la que se dirige. Se trata de un centro elitista, clasificado por algunos expertos como «Centro Conceptual». La «Galeria del Prado" por su parte, centro de moda y diseño, también es un centro elitista situado en la almendra, aunque fuera de las áreas comerciales: su clientela, también selecta, se encuentra, en una gran proporción dentro del área, en función de la proximidad del Congreso de Diputados, hoteles de lujo, el Museo del Prado, con un turismo internacional, y residencias de las clases altas madrileñas.

Característica casi general de los Centros Comerciales situados en la almedra, es la de no hallarse en edificio singular - salvo el caso del Mercado Puerta de Toledo, edificio rehabilitado como centro comercial de arte $\mathrm{y}$ antigüedades, $\mathrm{y}$ también Jumbo con su galeria comercial-, sino en el bajo y sótanos de un edificio dedicado a otros usos. El elevado costo del suelo y su escasez dentro del casco urbano, son determinantes del aspecto formal de estos centros.

Fuera de la almendra, los distritos periféricos de la ciudad de Madrid cuentan con un corto número de centros, si bien éstos tienen superficies mayores, por lo general, y se sitúan en edificios singulares. Asi, Alcampo Moratalaz con su galeria comercial, Arturo Soria Plaza, en el Distrito de Ciudad Lineal, La Florida y Sexta Avenida, pertenecientes ambos en la actualidad al Distrito de Moncloa, y el mayor de todos, Madrid-2, situado en la polémica Vaguada del Barrio del Pilar, en el Distrito de Fuencarral. Todos ellos vienen a paliar, en definitiva, deficiencias o carencias de equipamiento comercial y de servicios en la periferia de la ciudad, dentro del proceso descentralizador de equipamiento y servicios que se sigue hoy en la metrópoli madrileña.

Los Centros Comerciales existentes fuera del término municipal de la capital, se sitúan preferentemente en su entorno más inmediato, siendo las áreas más densamente dotadas el Sur y el Oeste.

El Sur metropolitano ha visto proliferar en los últimos años el número de Centros Comerciales, en función de su importante crecimiento y el volumen de población residente en la actualidad dentro del área. Los Centros Comerciales se extienden por Getafe, Leganés, Fuenlabrada, Parla, Móstoles y Alcorcón, enlazando, a través de los municipios del Suroeste -Boadilla del Monte y Villaviciosa de Odón, donde también proliferan los centros comerciales-, con los del Oeste: Las Rozas, Majadahonda y Pozuelo de Alarcón. Se constituye, asi, el área Sur-Suroeste-Oeste de la metrópoli madrileña, como el espacio de asentamiento preferente de los Centros Comerciales, dentro de la CAM. 
La tipologia de los centros varia, sin embargo, según el área de asentamiento, en función de la población residente: en el Sur, donde se concentra una elevada proporción de población industrial, la mayoría de los Centros Comerciales posee una poderosa "locomotora" de alimentación, en forma de hipermercado o supermercado. En muchos casos esta "locomotora" tiene el tamaño de un hipermercado, aunque no se puede clasificar como tal, por no contar más que con surtido de alimentación y drogueria, estando ausentes las secciones perfumeria, textil y bazar. Ello es frecuente en los centros de Móstoles.

En esta zona destaca Parquesur de Leganés, que se constituye como el mayor Centro Comercial de Europa, con un área de influencia de nivel regional y que ha venido a paliar las fuertes deficiencias de equipamiento comercial, de ocio y servicios de la zona Sur de Madrid, siendo un revitalizador importante de la misma.

La zona Oeste es, dentro del área metropolitana, la pionera en la implantación de Centros Comerciales. No debemos olvidar que el primer Centro Comercial de nuevo cuño de la Comunidad -en edificio exento proyectado con tal fin-, fue el "Zoco de Majadahonda", inaugurado en 1979 , que supuso un concepto revolucionario dentro de las formas tradicionales. El municipio empezaba a configurarse entonces como un área residencial en constante crecimiento, con una población de ingresos medios elevados, alto nivel de formación académica e importante proporción de jóvenes, rasgos que configuraban un estilo de vida muy marcado, equiparable a cualquier población de los alrededores de una gran ciudad europea. En aquel momento el Zoco solucionaba el déficit comercial y de equipamientos recreativos que presentaba un municipio de crecimiento rápido e intenso, dotando a éste de un lugar de encuentro civico-social, necesario y del que carecia.

A partir de entonces proliferan en todo el área occidental metropolitana, en los municipios en torno a la carretera N VI, con unas caracteristicas residenciales similares a Majadahonda - Aravaca, Pozuelo y las Rozas, singularmente-, los Zocos o Centros Comerciales de no muy amplias dimensiones, en edifico singular de dos plantas, por lo general, donde los locales comerciales tienen con frecuencia carácter de lujo y la "locomotora", caso de haberla, suele ser una mediana superficie de alimentación, sin carácter de discount. El dinamismo - que aún no se ha visto frenado- en la construcción de centros en la zona, se justifica por el continuo crecimiento de estos municipios, resultado de la "huida" de la ciudad, por parte de algunos sectores de la población, hacia zonas más abiertas y cercanas a la naturaleza. El poder adquisitivo de la población residente, es un factor que se halla en la base del fenómeno. 
Con todo, parece advertirse una cierta saturación de la oferta de este tipo de centros en la zona y un solapamiento en las distintas áreas de influencia: en torno a la N VI, por ejemplo, hay tres centros («Sexta Avenida", "Centro La Florida" y "Centro Comercial Las Lomas») a menos de 500 metros de distancia. Muy cerca de ellos, existe un proyecto en marcha, que englobará un Hipercor, locales, oficinas y una estación de ferrocarril, que se pretende actúe de foco centralizador disuasorio que evite en los posible la afluencia de público a la capital.

Fuera de estas áreas, los Centros Comerciales no son tan numerosos en la Comunidad de Madrid. Su distribución espacial está en función de la distribución de la población y de la red de carreteras nacionales, sobre todo. Asi, en el Corredor del Henares existen Centros Comerciales en torno a la carretera de Barcelona, en San Fernando y Alcalá de Henares. En torno a la carretera de Valencia, hay Centros Comerciales en Rivas-Vaciamadrid y Arganda. Junto a la carretera de Burgos, Alcobendas cuenta con un Centro Comercial.

Más allá del entorno metropolitano, en el área de la Sierra de Guadarrama, la "Sierra rica" de Madrid, existen dos Centros Comerciales en Collado Villalba, el municipio más poblado y que destaca en los últimos años como el centro industrial y de servicios más importante del área.

A lo largo de los párrafos anteriores han ido surgiendo algunos de los factores que explican la distribución espacial de hipermercados y Centros Comerciales en la CAM. En nuestro criterio, los más destacables son: la distribución de la población, la segregación social, la distribución de la red de carreteras, el planeamiento y el reparto y precio del suelo vacante.

1. La distribución de la población en la CAM explica, efectivamente, las líneas generales de la localización de las grandes superficies comerciales: éstas tienden a emplazarse en áreas donde existe una clientela potencial voluminosa. Asi, Madrid capital, donde se concentra el mayor número de hipermercados y Centros Comerciales, es el mercado más potente de la comunidad, con una población superior a los 3 millones de habitantes. Fuera de los límites de la ciudad, el Sur metropolitano destaca como un agregado de la misma con más de 1 millón de habitantes y una densidad comparable a la de la capital en municipios como Alcorcón, Móstoles, Leganés, Fuenlabrada y Parla. Esta concentración de población explica, sin duda, la proliferación de centros comerciales en la zona. Al Este de la capital, Coslada, San Fernando, Torrejón y Alcalá de Henares, forman una línea de asentamientos destacable, reuniendo algo más de un tercio de millón de habitantes y con densidades que individualizan el sector de su entorno. Al Nordeste destacan Alcobendas y San Sebastián de 
los Reyes, con poblaciones que superan los 70.000 y 50.000 habitantes respectivamente.

Al Norte y Oeste las aglomeraciones de población en torno a Madrid no son tan importantes. Al Oeste, la ocupación del suelo residencial es más extensiva, siendo, en general, asentamiento de clases sociales acomodadas. La zona, sin embargo, se halla en continua expansión, destacando por su población Pozuelo, Boadilla del Monte, Majadahonda y las Rozas. Las densidades de población son muy inferiores a las del municipio de Madrid, pero superiores a las de los municipios próximos.

Fuera del área metropolitana, destacan puntos aislados dentro de la Comunidad, que concentran volúmenes de población no tan elevados: Aranjuez, al Sur; Arganda al Sureste; Colmenar, al Norte y Collado Villalba (centro de la "Sierra rica»), al Oeste. Esta última zona se halla en expansión, no sólo como área de segundas residencias, sino también, en los últimos años, como zona de asentamiento definitivo, por parte de una población en muchos casos dependiente laboralmente de Madrid.

El área de influencia de la capital se amplia también aquí, jugando un papel fundamental la buena comunicación por ferrocarril existente.

2. La segregación social también explica algunas tendencias en la distribución espacial de Centros Comerciales e hipermercados, puesto que el dinero es, en definitiva, el primer y último motor del negocio comercial.

La polarización de las clases sociales en la CAM adquiere renovada importancia en los últimos años. La población de alto poder adquisitivo se traslada hacia el Oeste: el boom inmobiliario se manifiesta en la demanda de suelo urbanizado de mayor calidad en el eje de la carretera de la Coruña y la de El Escorial, que se prolongan con urbanizaciones residenciales y parques empresariales, creciendo de forma importante la renta media de la zona en los últimos años. Ello explica la proliferación de Centros Comerciales en una zona cuyo volumen de población no justifica el crecido y creciente número de ellos. En contraste, la polarización de las clases de menor poder adquisitivo en la corona Sur, el área industrial por excelencia, es patente.

Esta segregación no sólo explica el creciente número de Centros Comerciales en las áreas de residencia de las clases adineradas, sino también las importantes diferencias estructurales y formales de los mismos. Mientras en el Oeste los centros comerciales no poseen necesariamente una "locomotora" de alimentación, ésta es fundamental en los centros del Sur y tiene siempre carácter de discount. Asimismo, mientras el aspecto externo, la estructura arquitectónica y la decoración de los centros se ha- 
llan en un primer plano de interés en los implantados en el área occidental, estos aspectos se resuelven de forma más simple y económica en los de la zona Sur. Las firmas comerciales de los primeros centros son, con frecuencia, de élite; los altos precios, asimismo, actúan como reclamo en muchos casos y son selectivos del tipo de clientela a la que se pretende captar. En los centros del Sur, en contraste, los elementos de captación son exactamente los opuestos.

3. La distribución de la red de carreteras ha sido factor de incidencia en la localización espacial de hipermercados y Centros Comerciales, desde dos perspectivas diferentes. De un lado, por haber constituido el elemento decisivo en el tipo de crecimiento - estrellado- seguido por la metrópoli madrileña, correspondiendo, en general, los municipios de mayor crecimiento a los localizados en los bordes de las carreteras: al borde de la N.I, Alcobendas y San Sebastián de los Reyes; siguiendo la línea de la N. II, Coslada, San Fernando, Torrejón y Alcalá de Henares; junto a la N. III, Rivas-Vaciamadrid y Arganda; entre la N. IV, la carretera de Toledo y la N. V, Alcorcón, Leganés, Getafe, Móstoles, Fuenlabrada, Pinto y Parla; por último, la N. VI pasa por Las Rozas y junto a los municipios de Pozuelo y Majadahonda, siendo también factor impulsor de los pueblos de la Sierra rica que se hallan a sus bordes, singularmente Villalba.

De otro lado, conocemos la incidencia directa de las vias de comunicación en la implantación de los hipermercados y de algunos Centros Comerciales, que escogen localizaciones próximas a ellos para ampliar sus áreas de influencia, ya que el automóvil es el medio de transporte más generalmente utilizado por la clientela.

4. Planeamiento, reparto y precio del suelo vacante, son factores sin duda fundamentales a la hora de comprender la distribución de muchas de estas grandes superficies comerciales. La espectacular alza en el valor del suelo urbano en Madrid capital en la década de los 80 -efecto del creciente proceso de terciarización, el auge inmobiliario, la entrada de nuestro país en el Mercado Común, con la afluencia de capital extranjero, y la especulación sobre el suelo- tiene una fuerte incidencia en la estructura funcional de la ciudad, la expulsión de parte de la población (sobre todo población joven) y relocalización de ésta en su entorno y en la tendencia actual a la creación de un modelo de metrópoli policéntrica, que es apoyado por el planeamiento vigente.

Los criterios seguidos en el planeamiento urbanistico sobre dotación comercial, han experimentado un cambio reciente. Los Planes Generales dedicaban a esta función el suelo sobrante de ordenar los usos residenciales e industriales. Afortunadamente este planteamiento se ha superado y las necesidades de suelo para equipamiento comercial son contempla- 
das por el planificador. Este cambio de actitud obedece a la necesidad de amoldarse a las transformaciones producidas en la sociedad, como la progresiva diversidad de estratos sociales con estructuras y pautas de consumo diversas y la nueva distribución geográfica, todo ello enmarcado dentro de una estrategia urbana global, buscando el equilibrio entre una periferia fuerte, unos barrios que respondan a necesidades locales y el hipercentro ampliado y renovado que permita a los distribuidores situarse en todas y cada una de las áreas.

No obstante, hay una tensión de fondo entre el mundo de la planificación y el comercial, de muy difícil solución. La planificación es, por definición, conservadora. El comercio, por su parte, es estructuralmente dinámico porque tiene que responder a los cambios constantes de pautas de consumo. Esto hace que surjan nuevas fórmulas y provoca el desfase de las estructuras comerciales heredadas y un cambio vertiginoso en los comportamientos de localización.

En este sentido, el planificador podrá: a) forzar la evolución de comportamientos comerciales seguros, propiciando actividades bien localizadas y conectadas con las vias de transporte, como medio de difusión de mercancias y consumidores; b) dinamizar y revitalizar el tejido comercial degradado del centro urbano con operaciones más precisas; $c$ ) disminuir el riesgo de lo imprevisto, haciendo previsiones abiertas en determinadas áreas de territorio; o, por último, d) excluir el reglamentarismo y la zonificación rigida, creando mecanismos que faciliten la incorporación de cambios inesperados.

Concretando en el ámbito territorial que nos ocupa, en la CAM se están coordinando una serie de politicas económicas, sociales de transporte y de suelo, que permitirán la descongestión del centro, favoreciendo la creación de polos de servicios en zonas periféricas. Así, el centro, muy saturado, necesitará un proceso de descentralización y descongestión. La periferia municipal, sobre todo la zona Sur, bien dotada de espacios vacantes, irá expulsando de su territorio algunas actividades obsoletas (la industria pesada, por ejemplo), para dar cabida a otras comerciales y de servicios: en este contexto se ha de situar la construcción de Parque Sur, precisamente en esta zona, asi como la creación de una nueva universidad estatal, tres hospitales, un tanatorio, etc... De igual modo, la misma periferia Sur metropolitana y la periferia Este, con capacidad residencial y económica importante, recibirán un impulso institucional.

Ello se traduce en la creación y auge de focos de actividad metropolitana en distintos puntos fuera del centro consagrado de la ciudad y la creación de nuevas centralidades que absorban el choque de la fuerte demanda que sufre Madrid (CASTELLS, M. 1990). 


\section{ASPECTOS URBANISTICOS FORMALES}

Es imposible, en unas cuantas páginas, resumir los aspectos formales de los hipermercados y Centros Comerciales implantados en la CAM, tal es la diferencia y la riqueza de tipos y formas de los mismos. Por ello, y tratando de abarcar algunos de los aspectos más destacables, hemos seleccionado cinco centros que son de alguna manera ejemplos de tipos diversos: describiremos en las páginas siguientes un Parque Comercial que combina la oferta comercial con una potentísima oferta deportiva y de ocio (Parque Sur de Leganés), un Centro Regional de amplias dimensiones (Madrid-2), un pequeño Centro Comercial implantado en un área suburbana de clase alta (Las Lomas), un Centro de Moda localizado en un área comercial del interior de la ciudad (Multicentro Princesa) y un hipermercado con galería comercial (Pryca Pinar de las Rozas).

PARQUE SUR se emplaza en el término municipal de Leganés y supera, por sus dimensiones, cualquier otro proyecto realizado hasta hoy en España. Inaugurado en 1989, ocupa una superficie de $420.000 \mathrm{~m}^{2}$, de los que $110.000 \mathrm{~m}^{2}$ están dedicados a venta, 20.000 a almacenes y el resto a jardines y espacios deportivos y de ocio. Por su situación, es el centro de atracción de una amplísima zona comprendida entre las carreteras de Extremadura y Andalucia.

Se trata de un completo Parque Comercial con dos áreas claramente definidas: una comercial y otra puramente recreativa, integradas en un conjunto armónico en el que la combinación de zonas cubiertas con espacios al aire libre, parques y jardines con superficies de agua, crean un ambiente único.

El área comercial se ubica en una amplia y diáfana edificación de ladrillo visto, utilizado no sólo en las fachadas exteriores, sino también en los locales integrados en las diferentes calles temáticas. Este interior, unido a la proliferación de lucernarios y elementos de jardineria, confieren al lugar, a la vez que un aspecto de centro comercial tradicional, un sitio de paseo agradable y lleno de vida. La distribución en calles especializadas según los tipos de oferta comercial, facilita la orientación y su utilización por el comprador. Asi, se desarrollan la "Calle de la Moda", la "Calle del Hogar" y la céntrica "Calle del Comercio", en cuyo extremo se sitúa el Mercado, que consta de 28 puestos especializados de alimentación, al estilo tradicional. Las locomotoras del área comercial son un hipermercado de la cadena Alcampo de $11.000 \mathrm{~m}^{2}$ de s.v. y un gran almacén de la cadena Galerias Preciados, de parecida superficie. La oferta comercial se resume en 109 comercios ocasionales y 34 diarios. 
Al extremo de la Calle del Comercio se abre la «Plaza de las Barcas», donde se encuentran 8 salas de cine, dando paso a la zona propiamente recreativa del centro comercial. El «Paseo de la Isla» y el "Paseo del Lago" organizan la zona destinada a restauración, con bares y cafeterias, autoservicios, pizzerias, burgers, croissanterias, goffres y restaurantes especializados en diferentes cocinas nacionales e internacionales. Todo ello complementado con establecimientos de servicios, un hotel y un amplio espacio para terrazas en la zona que bordea el lago, formando uno de los espacios más acogedores del parque comercial.

El «Porche de las Oficinas» ocupa la fachada más próxima al aparcamiento y comprende más de 30 locales destinados a bancos, compañías de seguros, agencias de viajes y salones de convenciones, además de un edificio de oficinas. La «Plaza de Jauja», ocupada por bares y discopubs, cierra el Centro Comercial y da acceso a las diferentes instalaciones recreativas, cuya superficie supera los $300.000 \mathrm{~m}^{2}$. Su división por zonas facilita la localización de las diferentes instalaciones y la comunicación entre las mismas. Aqui se desarrollan una gran sala de conciertos y discoteca, un parque acuático y un parque de atracciones. El parque cuenta, asimismo, con $10.000 \mathrm{~m}^{2}$ de polideportivos - cubierto y descubierto- y un gimnasio. Al otro lado del espacioso parking, para 3.000 vehículos, y junto al acceso principal, se encuentra el “Área del Automóvil», con un Auto Center, una estación de servicio y un túnel de lavado.

Este ambicioso Parque Comercial ha venido a resolver, en gran medida, el déficit de equipamiento comercial, deportivo, de ocio y de servicios de la zona Sur de Madrid, siendo su área de influencia amplísima.

MADRID 2 abre sus puertas el 25 de octubre de 1983 en los terrenos conocidos con el nombre de “La Vaguada», junto al Barrio del Pilar, en el Distrito de Fuencarral, siendo el primer centro regional de la capital.

El proyecto es un alarde de arte e ingenio. Obra de José Ángel Rodrigo y César Manrique, consta de cuatro plantas, de las que dos se hallan enterradas, aprovechando el desnivel de la vaguada, configurando un parking con capacidad para 3.600 vehiculos. Las otras dos se hallan a nivel de la calle, formando el área comercial, que se remata con una terraza, zonas de ocio, donde la vegetación es el elemento decorativo fundamental. La estructura del edificio es rectangular, con fachadas revestidas de piedra natural, rodeadas de 345 jardineras colgantes dispuestas en escaleras que descienden desde la terraza hasta el nivel del suelo, ocultando a la vista del público la ronda de circunvalación, donde se sitúan los muelles de carga y descarga de mercancías. La vegetación funde toda la edificación. El conjunto se corona con veinticuatro velas blancas, que dan al centro un aire deportivo, al tiempo que matizan la luz que 
entra al interior por medio de unas lucernarias. En la decoración interior dominan los blancos en techos, paredes y suelos, con elementos decorativos vegetales, de agua y rocalla.

Madrid 2 está constituido por más de 300 establecimientos distribuidos en tres niveles y en torno a dos grandes locomotoras: Galerias Preciados y el hipermercado Alcampo. La Planta Baja es la de la compra cotidiana y el equipamiento personal; está compuesta por $10.000 \mathrm{~m}^{2}$ del hipermercado (nivel alimentario) y más de 60 puestos de una galería de alimentación tradicional, así como $6.000 \mathrm{~m}^{2}$ de Galerias Preciados, un almacén popular (C\&A) y unas 70 tiendas especializadas en todos los ramos del comercio. La Planta Alta está marcada por la especialización por zonas. En la parte Oeste predomina el Hogar, constituyendo "Artespaña" su principal polo de atracción. En el «mall» central se encuentra la "Plaza de los artesanos", un conjunto que integra 40 comercios especializados. Hacia el Este, Galerías Preciados tiene en esta planta $6.000 \mathrm{~m}^{2}$ y Alcampo 10.000 , dedicados a textil y hogar. Los $15.000 \mathrm{~m}^{2}$ restantes, repartidos entre un centenar de tiendas especializadas, dentro de las que destaca el "Centro de la Moda", con los mejores y más conocidos establecimientos, convierten a esta planta en la de la compra-placer.

La Planta Terraza se compone de una zona cubierta, en la cual están situados los restantes, bares, heladerias, etc..., asi como algunos comercios especializados y nueve salas de cine. La prolongación de esta zona hacia la última planta de Galerias Preciados está constituida por un parque con usos recreativos.

Como ocurre con Parque Sur, Madrid-2 ha venido a paliar en gran medida el déficit comercial y de servicios de un área de Madrid, constituyéndose como centro de una amplia zona al Norte de la capital.

La CIUDAD COMERCIAL LAS LOMAS toma su nombre de la urbanización donde está ubicada, perteneciente al municipio de Boadilla del Monte. Es una zona residencial de clase alta, en la que predominan las urbanizaciones de lujo y las viviendas unifamiliares. Su promotor fue el grupo financiero Alfaro y se abrió al público el año 1990. El alto valor del suelo en la zona y la calidad de la construcción, encareció enormemente el precio de los locales, siendo, en consecuencia, la oferta claramente elitista, con firmas de calidad reconocida y altos precios, en consonancia con la demanda a la que va dirigida.

La Ciudad Comercial ocupa una superficie aproximada de $29.000 \mathrm{~m}^{2}$, de los que 14.000 son jardines urbanizados y cuidados con todo detalle, que circundan el Centro Comercial. De los $15.000 \mathrm{~m}^{2}$ construidos, 7.000 se reparten en 129 locales, dedicados en su mayoría al comercio ocasional (43). Del resto, nueve son de restauración, siete ocio, nueve otros servi- 
cios y tres comercio diario. La mayor superficie comercial es la ocupada por el Supermercado Alfaro.

El edificio consta de tres alturas más dos niveles subterráneos de parking, con 486 plazas. Concebido arquitectónicamente como un gran cubo revestido de granito pulido - lo que le confiere un aire lujoso-, su nota característica es el equilibrio entre la modernidad, la elegancia, lo suntuoso y la funcionalidad. El interior, ordenado y luminoso, se estructura en torno a dos plazas dotadas de lucernarios y circundadas por suaves rampas, sobre las que se sitúan los diferentes locales. La decoración está minuciosamente cuidada: mármoles y cristales conjugan con dorados y espejos y combinan con el verdor de los ajardinamiento, en un ambiente refinado.

MULTICENTRO PRINCESA es un Centro de Moda localizado dentro de un área comercial del interior de Madrid, próximo, por otra parte, a un gran almacén de la cadena El Corte Inglés. Como tal centro de moda, está constituido por un número de establecimientos comerciales minoristas dedicados todos ellos a vestido, calzado y complementos. No posee «locomotora" alguna, ni ningún tipo de local dedicado al ocio, de manera que la oferta comercial únicamente se complementa con una reducida oferta de restaruación: una pequeña cafetería y un burger, que abrirá sus puertas próximamente. Su poder de atracción como Centro Comercial se basa en la agrupación de minoristas del mismo sector de actividad y su inclusión dentro de un área comercial consagrada de la ciudad.

Abierto en 1976, el Centro pertenece a una cadena que cuenta con dos empresas más dentro de la capital, de iguales caracteristicas y emplazadas estratégicamente, también dentro de áreas comerciales urbanas: Multicentro Serrano y Multicentro Orense.

Multicentro Princesa ocupa las plantas baja y sótano de un moderno edificio de siete alturas construido en línea de fachada dentro de una manzana de edificación cerrada. Sus $4.300 \mathrm{~m} 2$ de superficie construida se desarrollan en un espacio aproximadamente rectangular, que atraviesa longitudinalmente el fondo de la manzana, poseyendo el centro dos accesos, por calles opuestas, y aprovechando en su interior la luz natural que penetra através del patio de luces del edificio. El eje de este patio de luces marca el centro del espacio comercial, donde se encuentra un amplio hueco, con la escalera mecánica de comunicación entre ambas plantas. La decoración interior se basa en la línea quebrada que describen las fachadas de los locales y las columnas de espejo que soportan la estructura.

Los $2.500 \mathrm{~m} 2$ de superficie útil con que cuenta el centro se distribuyen en 56 locales comerciales del sector moda y dos locales de restauración. 
Muchas de las firmas instaladas son conocidas, si bien los precios de los articulos, en general, están en consonancia con el área comercial donde el centro se inscribe y con el tipo de clientela, joven, a la que se pretende captar.

EL CENTRO COMERCIAL PRYCA EL PINAR DE LAS ROZAS es representativo de un tipo de gran superficie desarrollado durante la primera fase de expansión comercial en España (1973-84).

Construido en 1981, fuera del núcleo urbano de Madrid, Pryca se halla dentro de una amplia zona residencial suburbana en continuo crecimiento y junto a una carretera nacional (N. VI) que es, a la vez, vía de acceso a la Sierra madrileña y eje de tránsito diario por el movimiento pendular de la población.

Se trata de un solar de más de $85.000 \mathrm{~m} 2$, donde se desarrolla un amplisimo parking (2.137 plazas) y el edificio del centro comercial, que consta de $8.800 \mathrm{~m} 2$ de venta y 7.300 de almacén. La mayor parte de la superficie de venta corresponde al hipermercado Pryca (7.300 m2), eje y motor del centro comercial. Fuera del edificio, en los extremos de la explanada del parking, se hallan dos servicios del automóvil, establecimientos que no pertenecen a la empresa Hipermercados Pryca S.A.

El espacio edificado es una nave rectangular de estructura simple y materiales prefabricados, donde el único elemento decorativo es el remate de la puerta principal de acceso. El resto de la fábrica ofrece un aspecto sobrio y funcional, quizá como reclamo al carácter de discount del establecimiento. El edificio se resuelve interiormente en una $T$ invertida, donde el eje vertical sería la superficie de venta del hiper - a cuyos lados se desarrollan los almacenes-y el horizontal la galería comercial, dispuesta en dos bandas, una exterior, de cara al parking, y otra interior, que forma un frente de locales que dan a las cajas de salida del hipermercado. Sobre los locales interiores se sitúa toda una galeria de oficinas. El interior del hipermercado se resuelve en pasillos horizontales y verticales, donde las zonas de alimentación, perfumeria-drogueria, confección y bazar, se hallan perfectamente diferenciadas. En cuanto a los locales que componen la galería comercial, 24 en total, 12 son de comercio ocasional, cuatro de comercio diario aunque especializado, cuatro de restauración y cuatro de otros servicios.

Los planes urbanisticos a corto plazo dotan al centro de buenas perspectivas, con la construcción de la carretera de circunvalación a Madrid M-40 -que bordeará el centro, favoreciendo su accesibilidad-y la instalación de un parque empresarial, ya en construcción, al otro lado de la carretera - lo que aumentará la cuota de mercado-. Las buenas perspectivas se han plasmado en una ampliación del edificio, proyectada para 
el próximo año, a costa del parking, aumentando en superficie y número de locales la galeria comercial.

\section{5) CONCLUSIÓN: IMPACTO DE LAS GRANDES SUPERFICIES EN EL ENTORNO.}

Madrid ha experimentado en los últimos años transformaciones semejantes a las de las grandes ciudades de Europa Occidental y Norteamérica. La expansión de la ciudad y la elevación del nivel de vida, han dado lugar a un reciente proceso de descentralización, con la creación de nuevas áreas comerciales y de servicios, localizadas fundamentalmente en la periferia y orla periurbana. Dentro de estas transformaciones, Hipermercados y Centros Comerciales han tenido un papel protagonista, produciendo un fuerte impacto en las áreas donde se implantan.

Entres sus efectos beneficiosos, queremos destacar la mejora que suponen en el equipamiento comercial y recreativo de las zonas donde se ubican, constituyendo la única oferta en áreas muy deficitarias y diversificándola en las demás. Además, en sectores que han nacido de espaldas a la racionalidad planificadora, los centros comerciales integrados actúan como verdaderos centros de atracción de la población, ofreciendo un lugar de relación que viene a suplir la falta de espacios urbanos con esta función.

En el caso de grandes centros planificados, sus proyectos contemplan la creación y mejora de las vias de acceso (Parque Sur, Madrid 2...), con lo que toda la zona se ve favorecida. Asimismo, existe una repercusión urbanistica sobre el suelo residencial de las zonas inmediatas, que se revaloriza, mejorando la calidad arquitectónica.

Por último, un conjunto comercial de gran superficie supone en muchos casos una fuente de puestos de trabajo para su área circundante. En definitiva, con frecuencia la implantación de un Centro Comercial se traduce en una revitalización económica de su área circundante.

Pero no todos los efectos son beneficiosos. Al ser Hipermercados y Centros Comerciales focos de atracción mercantil, recreativa y social, son efecto y causa a la vez de transformaciones en los hábitos de consumo y ocio. En este sentido, donde más se ha dejado sentir el efecto de las grandes superficies es en el comercio detallista de su entorno. La racionalidad y el concepto de ahorro en tiempo y dinero que los hipermercados crean en el comprador, hace que los pequeños comercios tradicionales de alimentación, droguerias-perfumerias, equipamiento personal y equi- 
pamiento del hogar pierdan clientela. El efecto se deja sentir también en autoservicios y supermercados. El comercio especializado de uso ocasional no sufre tan fuerte impacto, llegando a beneficiarse si su situación es próxima a la gran superficie, por la mayor afluencia de público, y perjudicándose, en líneas generales, si su situación no es suficientemente cercana (Estudio de Mercado y Opinión. Consultora Emer. CAM, 1991).

Otros efectos comerciales que hipers y Centros Comerciales tienen en su entorno, son la congestión y el aumento de los indices de contaminación acústica y medioambiental que su poder de atracción y el creciente tráfico generan en las horas, dias y fechas de mayor afluencia de compradores. Esto es más notorio en aquellos centros localizados dentro del término de Madrid y menos grave en el caso de los hipermercados situados al borde de importantes vias, dado que el dia e intervalo de mayor afluencia de público a ellos (sábados por la tarde) coincide, de una manera general, con el espacio de tiempo que las vias soportan menos tráfico.

Al margen de los efectos positivos o negativos de Hipermercados y Centros Comerciales en su área inmediata, no cabe duda de que ambas fórmulas han supuesto una renovación radical en la estructura comercial de la comunidad, igual que ha ocurrido en otras comunidades españolas y fuera de nuestro pais, en Europa Occidental y Norteamérica.

Como colofón, señalar que la actividad comercial en la sociedad actual es una de las más dinámicas; constantemente aparecen nuevas formas comerciales, tanto desde la aparición de nuevas tipologias, como desde el punto de vista de la organización empresarial. Todo esto hace muy dificil acometer una planificación rigida, que en la mayoria de los casos queda ya obsoleta antes de entrar en vigor.

No obstante, es de agradecer la iniciativa de la CAM de articular instrumentos de estudio de las estructuras comerciales madrileñas desde la doble perspectiva espacial y social. Son los Programas de Actuación Comercial, los PAC. El objetivo de estos programas es la formulación de propuestas de actuación que sirvan de base a las entidades públicas y privadas a la hora de acometer la implantación de nuevos equipamientos comerciales, en orden a la racionalización y modernización del sector comercial dentro de la CAM.

Bien es cierto que este esfuerzo no ha satisfecho totalmente los objetivos iniciales. De aqui la importancia de establecer cauces dinámicos de participación de todos los sectores implicados: empresarios, Cámara de Comercio, instituciones de la Comunidad, asociaciones de comerciantes, grandes cadenas, etc..., para lograr compromisos serios en aras a la mejor adecuación futura de estos equipamientos, de acuerdo con distintos 
parámetros: demográficos, socioeconómicos, de nivel de dotación, capacidad de gastos, urbanisticos, etc...

\section{BIBLIOGRAFIA}

Actas del Primer Congreso Español de Centros Comerciales, "Los Centros Comerciales en España». Octubre 1988, 16 págs.

ACTUALIDAD ECONÓMICA, 6 de marzo de 1989: “Todo bajo un mismo techo", págs. 84-87.

ARAL, 10 de septiembre de 1988: "El Centro Comercial: entre el urbanismo y la sorpresa", págs. 37-39.

ARAL, 20 de mayo de 1989: IV Jornadas de distribución comercial. "Supermercados, nuevas experiencias europeas".

ARAL, 16 de septiembre de 1989: "Pryca. Buenos resultados en 1988".

ARAL, 28 de octubre de 1989: "Fuerte desarrollo de Hipercor», págs. 17-18.

Bodega, I. y Lora-Tamayo, G. (1990): "El Centro Comercial Madrid-2. La Vaguada». Primeras Jornadas de Geografia y Comercio. Grandes Superficies Comerciales. Madrid, págs. 368-394.

Castells, M., Leal, J., Leguina, J. y Naredo, J. M. (1990), “¿De qué Madrid hablamos? 1. Crisis de crecimiento». Alfoz núms. 74-75, págs.

Castresana, J. (1976): “Efectos Comerciales y Urbanisticos de los hipermercados de BarceIona". I.C.E., febrero 1976 , págs. 86-99.

COMUNIDAD DE MADRID (1991): La influencia de las grandes superficies comerciales en la Comunidad de Madrid. Consejeria de Economia. Col. Estudios y Análisis, 101 págs.

ComunidAD DE MADRID (1991): Jornadas sobre comercio y territorio. Criterios de dotación y localización. Consejeria de Economia. Colección Estudios y Análisis. 127 págs.

ComuniDAD DE MADRID (1991): Guia de grandes superficies comerciales de la Comunidad de Madrid. Consejeria de Economia, 88 págs.

CheCA SÁnCHEZ, A. (1975): Estructura Comercial de Madrid. Estudio geográfico de Grandes Almacenes. Tesis Doctoral inédita. Facultad de Geografía e Historia. Universidad Complutense de Madrid. 3 tomos.

Checa Sanchez, A. (1989): “Teorias y métodos de la Geografia del sector comercial», Actas del II Congreso de Teoria y Metodología de las Clencias.

Checa Sanchez, A. (1990): "Repercusiones de las Grandes Superficies Comerciales en otras formas de comercio". Ponencia en Primeras Jornadas de Geografia y Comercio: Grandes Superficies comerciales. Madrid, págs. 265-286.

DINERO, 23 de octubre de 1987: "Madrid: La montée en puissance des centres commerciaux". págs. 50-51.

Dinero, octubre 1990: "Los Centros Comerciales, último grito en ventas", págs. 34-36.

Dirección Construcción, enero 1990: "Ocio y Comercio, un matrimonio feliz": Entrevista a L. M. Martín Romero, Vicepresidente de Parques Urbanos.

Distribución Actualidad, octubre 1987: "El comprador del Hipermercado", págs. 127-134.

DistriBución ACTUALIDAD, octubre 1988: "La gran aventura del discount. 25 años de hipermercado en Europa».

Distribución Actualidad, octubre 1988: "Poker de Ases. Las empresas de hipermercados".

DistRIBUCIÓN ACTUALIDAD, octubre 1988: “El Hiper en versión Nielsen», pág. 16.

Distribucion ACtuAlidad, octubre 1989: "Las empresas de Hipermercados. Acortar distancias", págs. 137-148.

Distribucion Actualidad, febrero 1990: "Cara a cara: Fernando Ramos, el "hombre Zoco", págs. 33-39.

Fernandez, J. (1989): “Mercado Puerta de Toledo, una oferta elitista". Distribución Actualidad, octubre 1989, págs. 29-35. 
Folletos publicitarios de distintos Centros Comerciales.

FriAs, M. (1983): “Bello y útil. La Vaguada (España)». Nueva Empresa, octubre 1983, págs. 16-19.

Le Blanc, M. (1983): «Madrid-2, une mine d'or dans un ravin?». Points de Vente, diciembre 1983. Paris, págs. $42-46$.

Lora-Tamayo, G. y Bodega, 1. (1990): "Primera nota sobre grandes superficies comerciales en la ciudad de Madrid". Primeras Jornadas sobre Geografia y Comercio: Grandes Superficies Comerciales. Madrid, págs. 164-189.

MARICOURT, R. (1989): "La nueva revolución en la distribución: del Hipermercado al Hiperservicio», Opinión, 1989, págs. 22-24.

MeACADo, enero 1988: "Centros Comerciales en Madrid. ¿Saturación o demanda?», págs. 1117.

MERCADO, febrero 1988: "Los March amplian la tienda", págs. 48 y ss.

MERCADO, julio 1989: "Los hipermercados están de moda", págs. 57-59.

Ministerio de Economia y Hacienda (1991): Centros Comerciales. Mercados Minoritas. Informe y Directorio. Dirección General de Comercio Interior. Colección Estudios 41, 2 vols.

PuLIDO, A. (1976): Caracteristicas e implicaciones de los hipermercados". ICE, febrero 1976, pàgs. $71-81$.

Rios, A., CHeCA, A. y Palacios, T. (1990): “Los nuevos Centros Comerciales como respuesta a un nuevo estilo de vida. Su plasmación en la Comunidad Madrileñan. Primeras Jornadas de Geografia y Comercio: Grandes Superficies Comerciales. Madrid, págs. 216234.

Ríos, J. y LEAL, J. (1988): Los espacios colectivos en la ciudad. Ed. MOPU-ITUR. Madrid.

RocA, J. A. (1984): "Todos hacen negocio". Actualidad Económica, noviembre 1984, págs. 117-119.

RANKING, enero 1989: El imperio de las grandes superficies, págs. 64-77.

SANCHEZ DEL, RIO, R. (1988): “Centros Comerciales Tradicionales". Primer Curso de Urbanismo Comercial. COAM.

Sempere, M. A. (1989): “Los Hipermercados revolucionan la distribución española». El Nuevo Lunes, $n .^{\circ} 19$, enero 1989.

SoriA, M. (1987): “Informe especial sobre grandes superficies". El Comestible, $n .{ }^{\circ} 348$, marzo, 1987, págs. 5-32.

TARILONTE, E. (1989): “En España se han instalado 79 hipermercados desde 1973». Financial Food, marzo 1989. 


\section{CUADRO 1. CENTROS COMERCIALES E HIPERMERCADOS EN LA COMUNIDAD DE MADRID}

A) MUNICIPIO DE MADRID

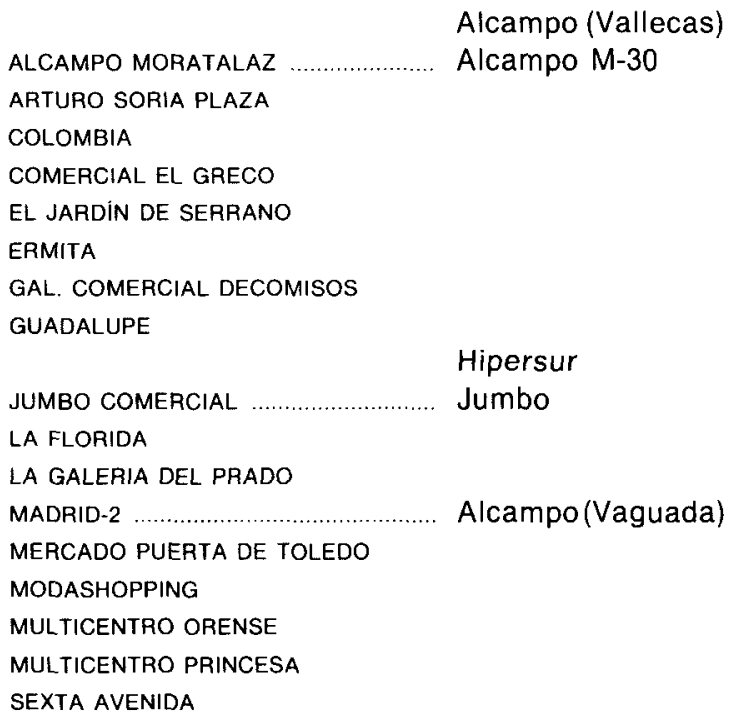

B) RESTO DE LA CAM

Municipio

ALCALÁ DE HENARES

EL VAL

LA DEHESA

Alcampo

ALCOBENDAS

BULEVARO

CONTINENTE

Continente

ALCORCON

Ecomix

Hipercás

S. JOSE DE VALDERAS

Hipercor

ALGETE SANTO DOMINGO

ARGANDA DEL REY ZOCO ARGANDA

BOADILLA DEL MONTE CENTRO COMERCIAL LAS LOMAS CIUDAD COMERCIAL LAS LOMAS ZOCO BOADILLA

COLMENAR VIEJO EL MIRADOR

COLLADO VILLALBA CANGURO ZOCO VILLALBA

COSLADA ZOCOSLADA EUROPA

FUENLABRADA I

Preco 


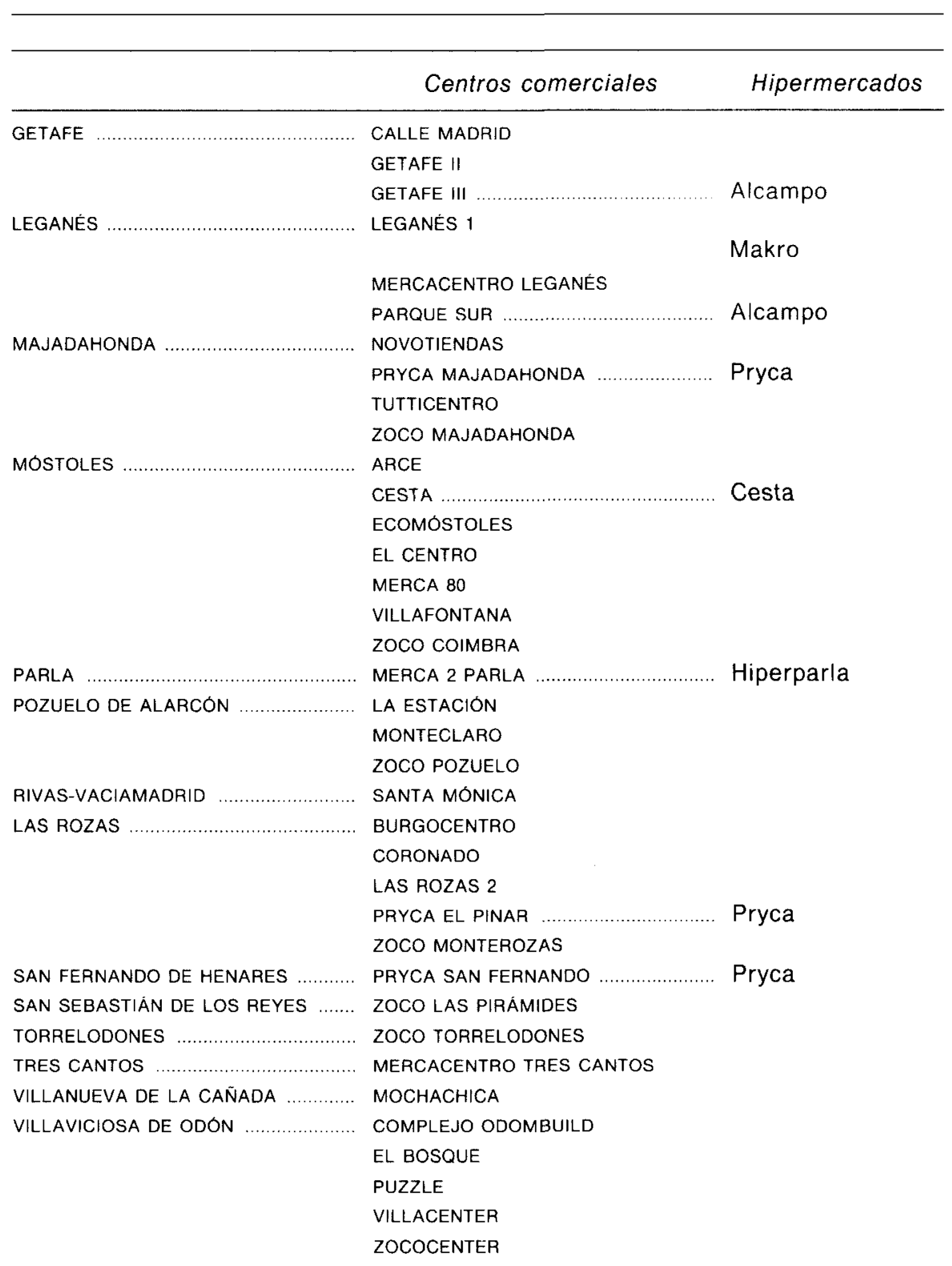

